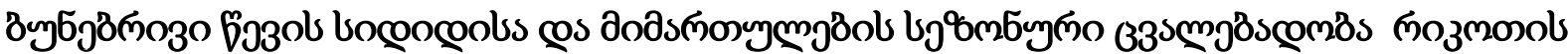

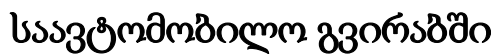

m. ms6hşs 6. Әymmsdo

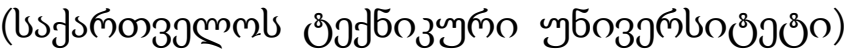

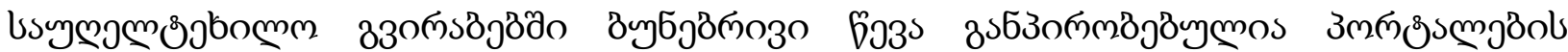

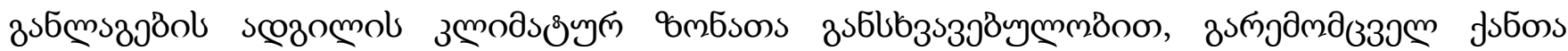

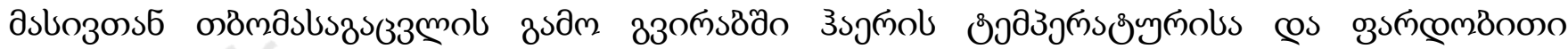

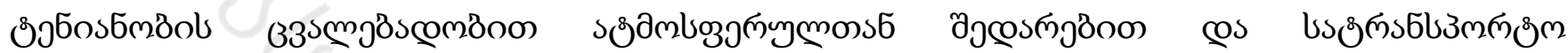

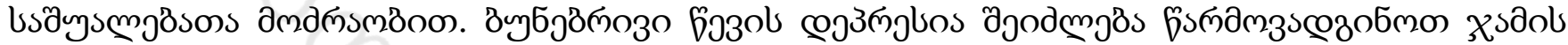
usboom

$$
h_{n}=h_{p}+h_{t, \varphi}+h_{t r}
$$

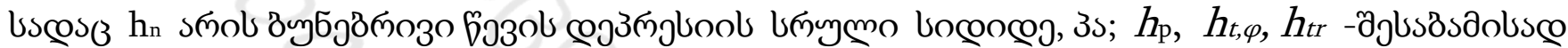

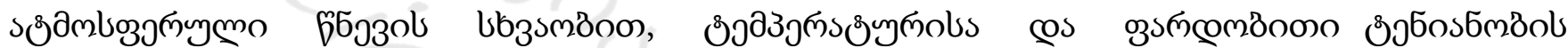

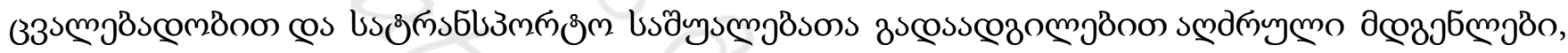
35.

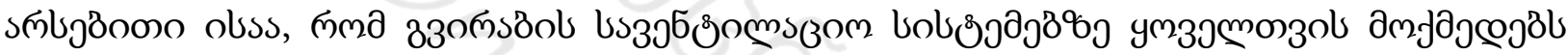

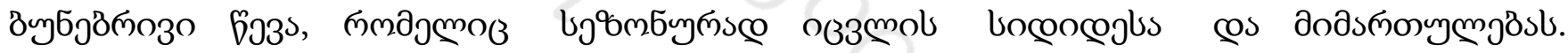

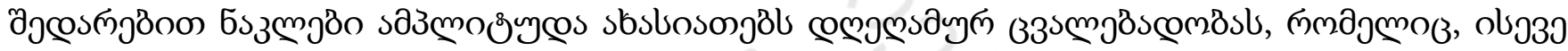

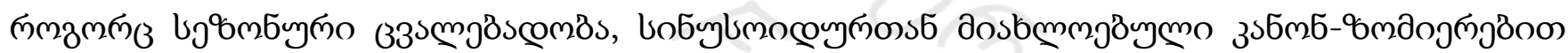
bubosongos.

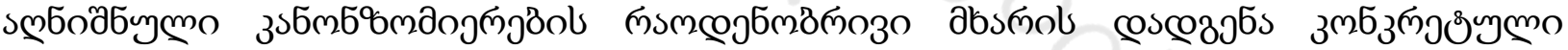

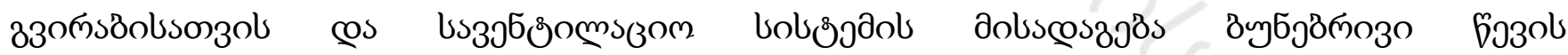

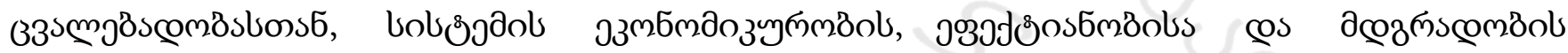

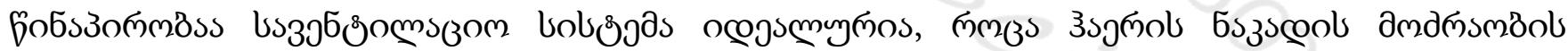

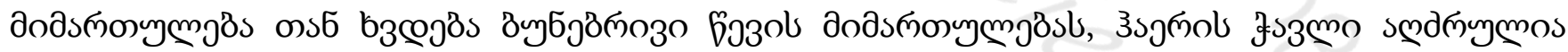

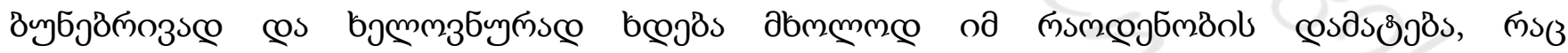

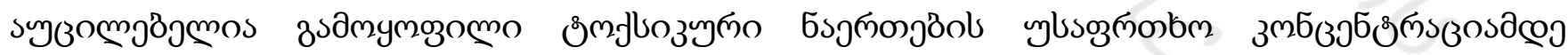

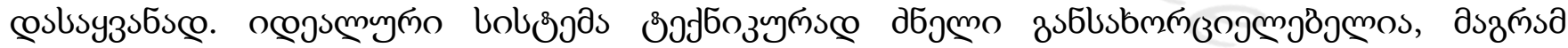

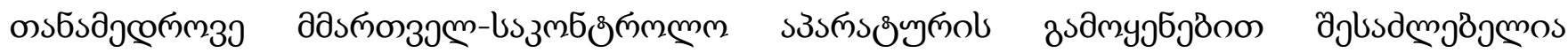

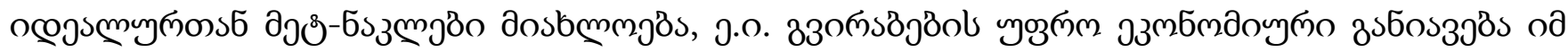

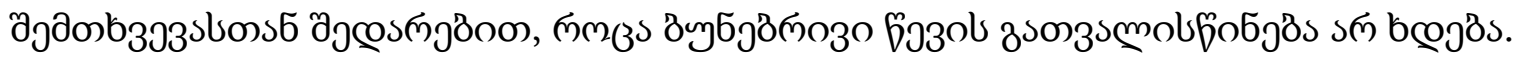

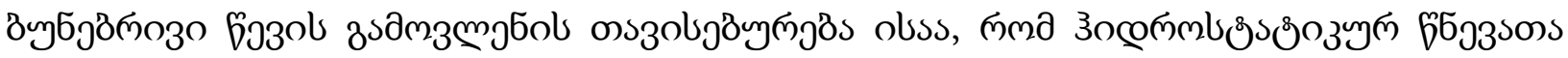

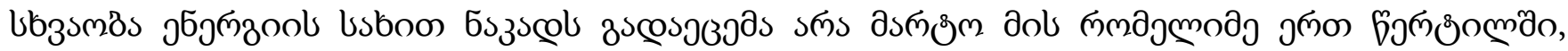

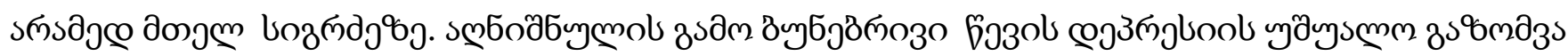

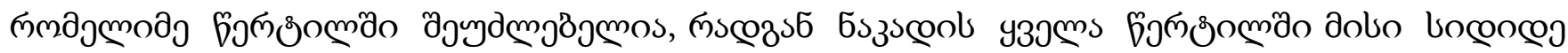

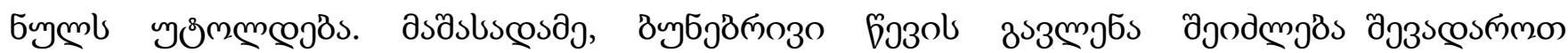

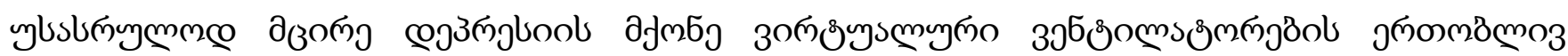




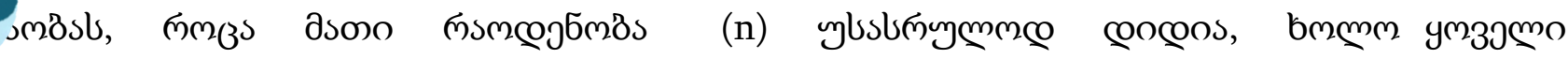

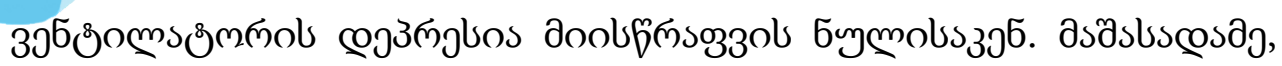

$$
h=\frac{h_{n}}{n} \text {, }
$$

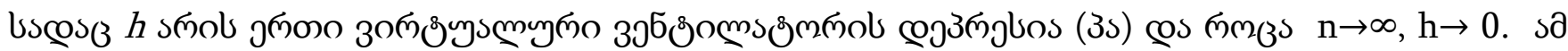

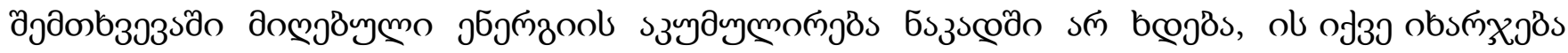

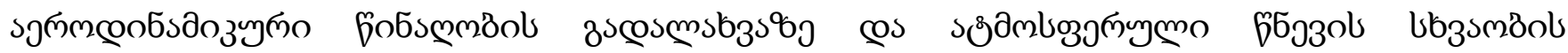

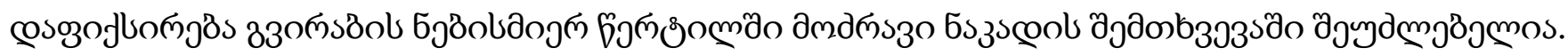

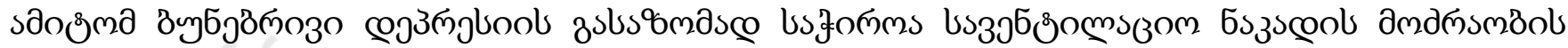

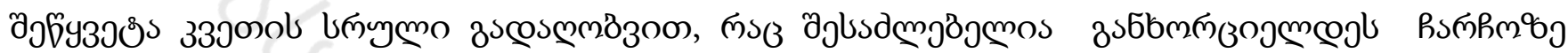

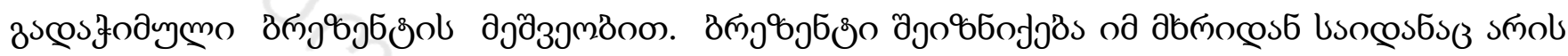

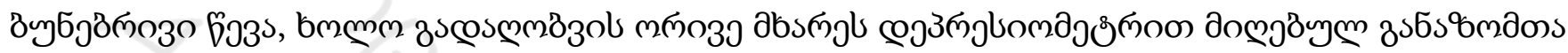

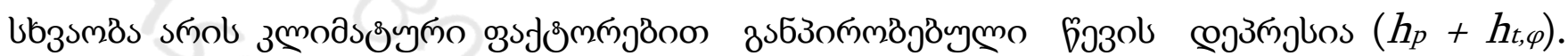

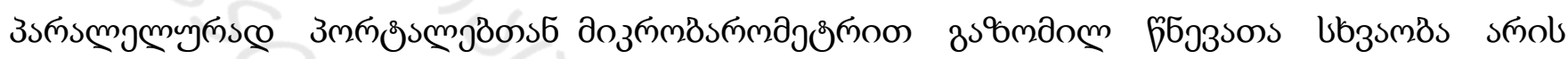

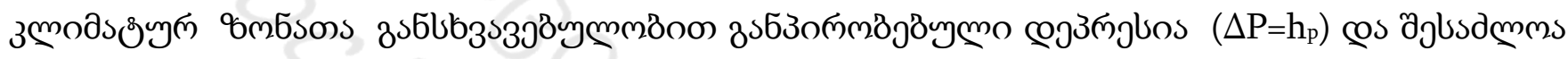

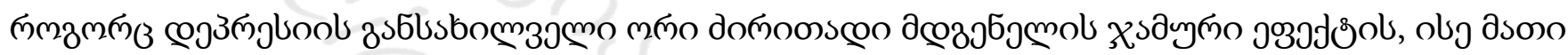

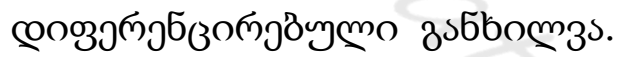

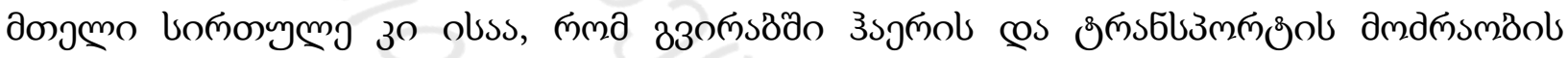

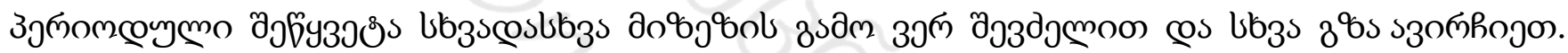

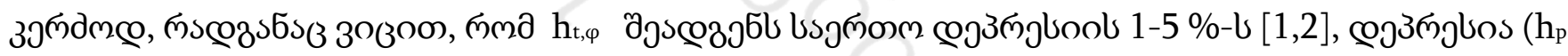

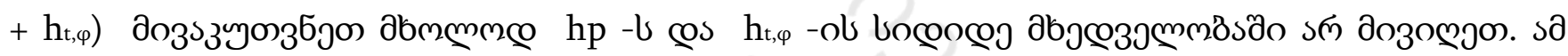

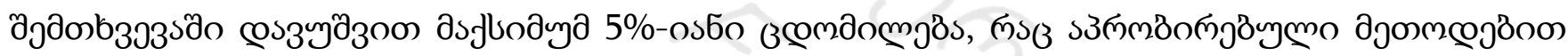

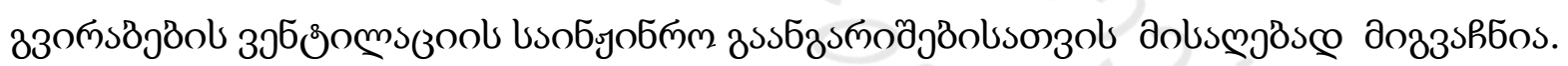

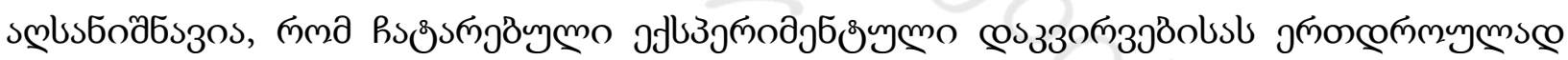

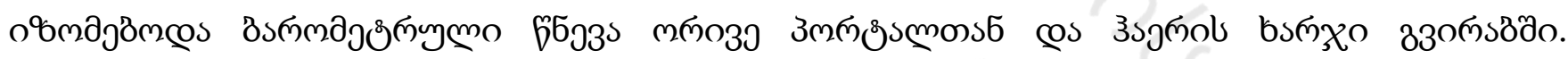

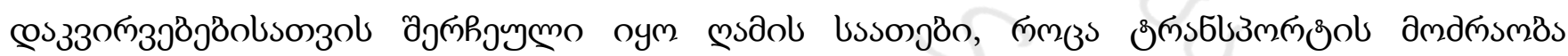

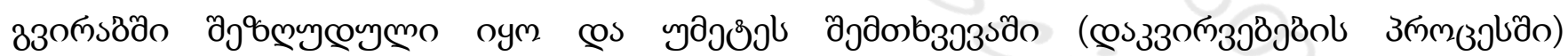

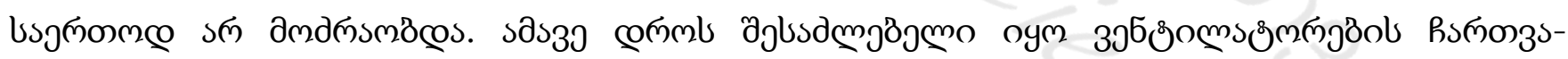

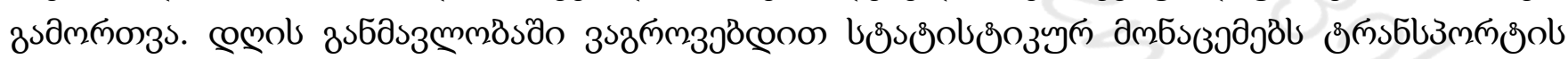

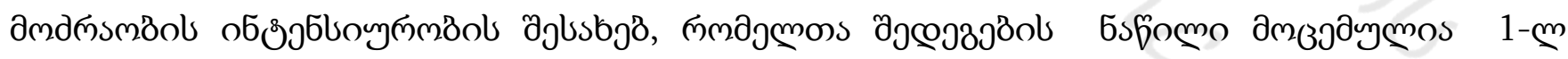

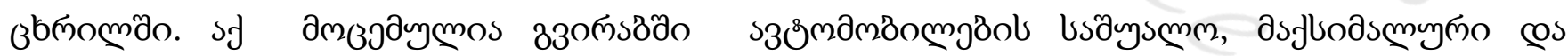

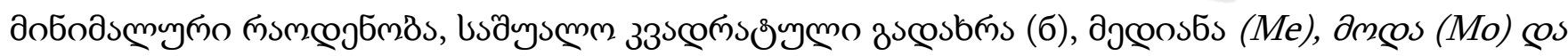

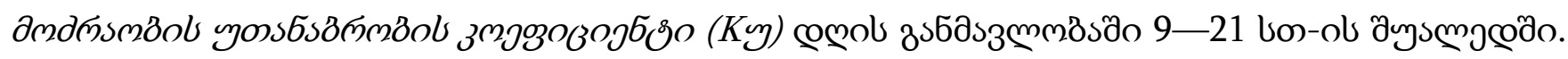

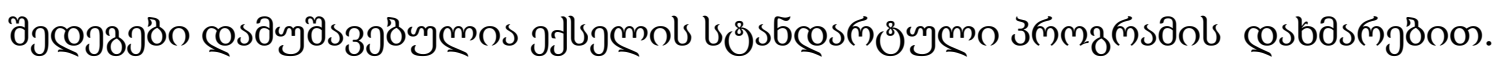

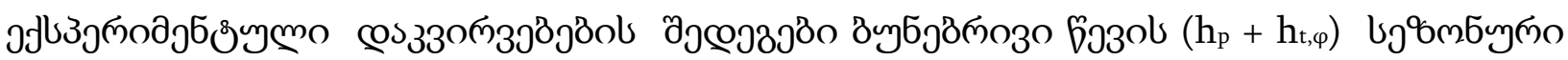

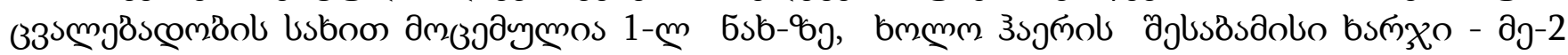

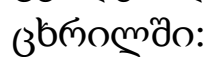

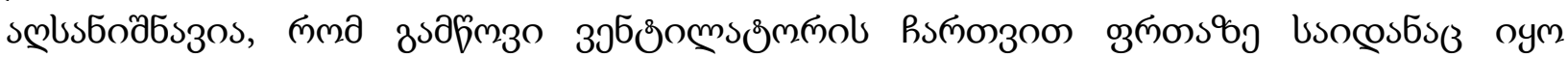




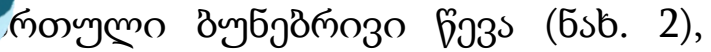

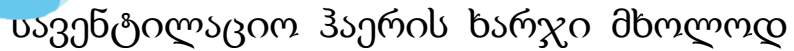

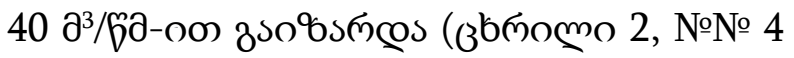
cos 8).

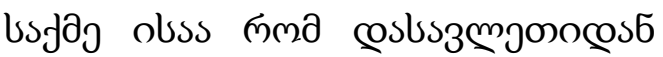

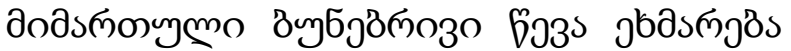

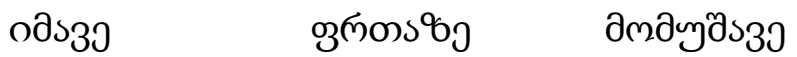

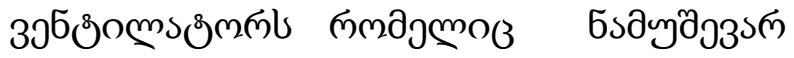

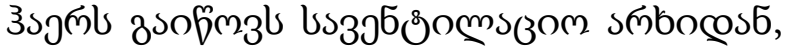

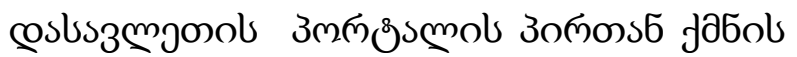

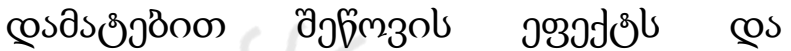

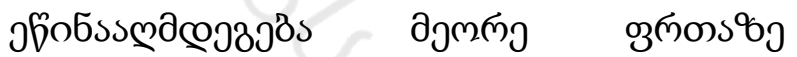

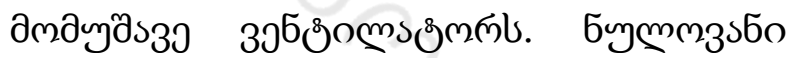

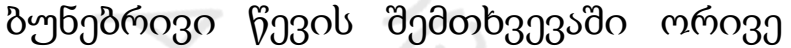

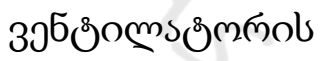

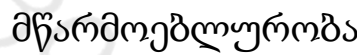

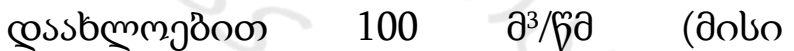

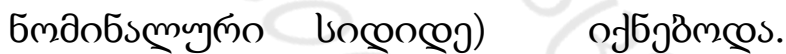

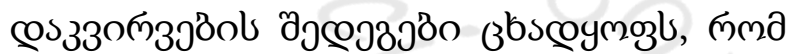

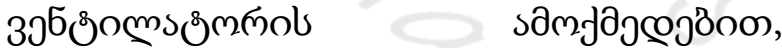

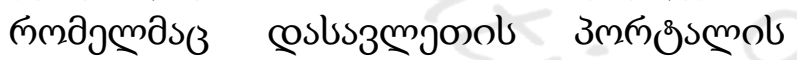
uszsm 5sfomano cosas

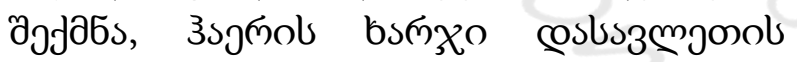

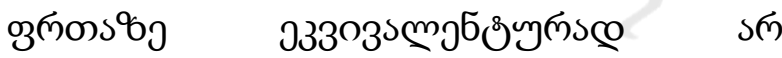
zso\%smes. saol do\%g\%se doz3sh6os

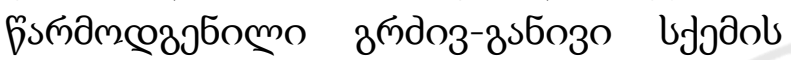

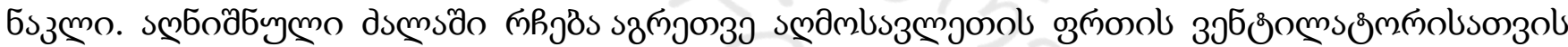

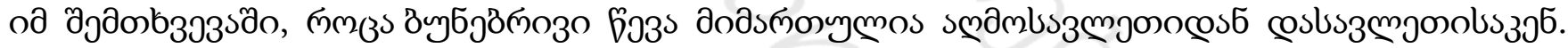

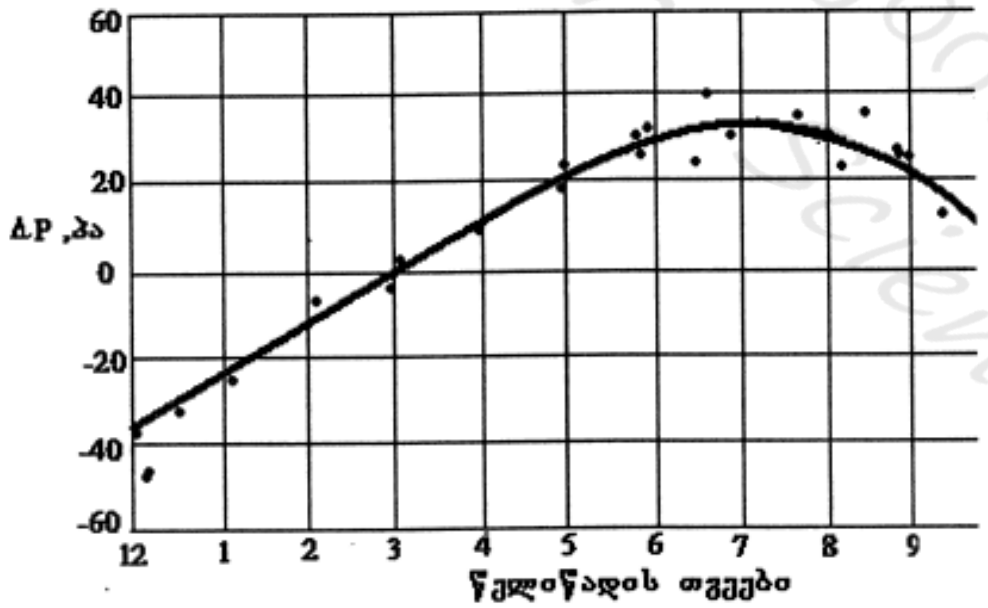

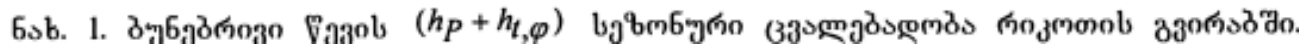

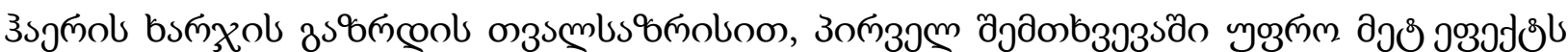

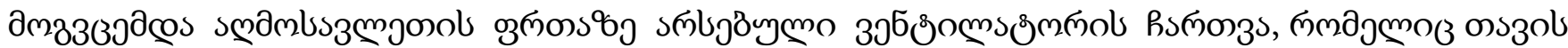

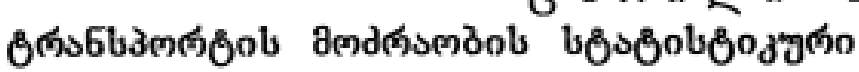

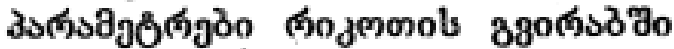

\begin{tabular}{|c|c|c|c|c|c|c|c|}
\hline \multirow{2}{*}{$\underset{\mathrm{E}}{\mathrm{E}}$} & \multicolumn{3}{|c|}{ 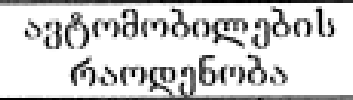 } & \multirow[b]{2}{*}{$\sigma$} & \multirow[b]{2}{*}{$\mathrm{Me}$} & \multirow[b]{2}{*}{ Mo } & \multirow[b]{2}{*}{$\mathrm{K}_{\text {-y }}$} \\
\hline & 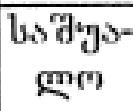 & कs jls. & 2o6. & & & & \\
\hline \multicolumn{8}{|c|}{ 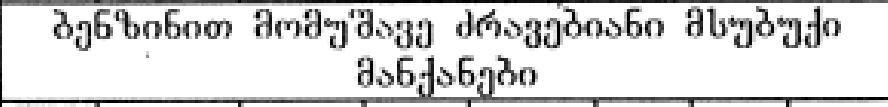 } \\
\hline 1986 & 263 & 480 & 118 & 118,3 & 252 & - & 2,35 \\
\hline 1997 & 277 & 334 & 219 & 35,6 & 274 & - & 1,39 \\
\hline 2004 & 261 & 326 & 194 & 45,0 & 252 & 241 & 1,52 \\
\hline \multicolumn{8}{|c|}{ 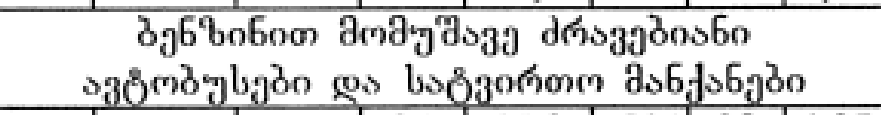 } \\
\hline 1986 & 52 & 81 & 25 & 15,9 & 51 & 38 & 1,37 \\
\hline 1997 & 55 & 92 & 29 & 16,1 & 50 & 50 & 1,35 \\
\hline 2004 & 52 & 66 & 38 & 9,0 & 56 & 39 & 1,52 \\
\hline \multicolumn{8}{|c|}{ 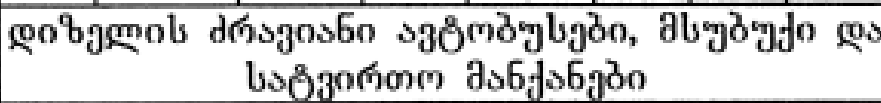 } \\
\hline 1986 & 21 & 35 & 13 & 6,2 & 21 & 13 & 1,90 \\
\hline 1997 & 23 & 33 & 17 & 4,6 & 25 & 25 & 1,60 \\
\hline 2004 & 50 & 61 & 43 & 6,0 & 51 & 44 & 1,36 \\
\hline \multicolumn{8}{|c|}{ 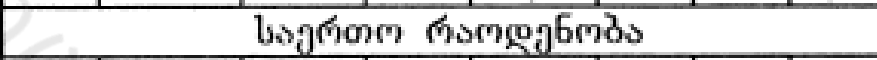 } \\
\hline 1986 & 336 & 589 & 165 & 130,1 & 324 & - & 2,17 \\
\hline 1997 & 355 & 400 & 281 & 38,4 & 359 & 400 & 1,32 \\
\hline 2004 & 364 & 425 & 277 & 51,0 & 344 & 423 & 1,42 \\
\hline
\end{tabular}




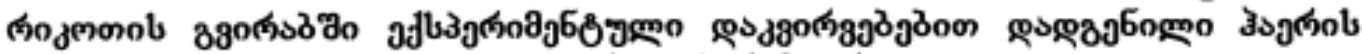

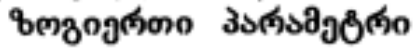

\begin{tabular}{|c|c|c|c|c|c|c|}
\hline $\mathrm{N}$ & कstrogen & 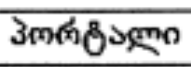 & $\mathrm{v}, \mathrm{a} / \mathrm{pa}^{-}$ & $\mathrm{Q}, \mathfrak{P} / \mathrm{P}^{2}$ & $\Delta P, 3 \mathrm{~s}$ & 6s \\
\hline 1 & 22.0997 & spamb. & 2,14 & 128,4 & 252 & galuagenongs6 \\
\hline 2 & 23.09 .97 & spaml. & 2,11 & 126,6 & 24,5 & gulsasengongas \\
\hline 3 & 23.09 .97 & golisgen. & 2,05 & 123,0 & 23,1 & gulajangonogs6 \\
\hline 4 & 23.09 .97 & gstosam. & 2,71 & $162,6^{*}$ & 23,1 & pslazengonogs \\
\hline 5 & 04.12 .03 & spdml. & 2,07 & $124,4^{\text {* } k \text { * }}$ & $-21,1$ & sedmls jen josocos 6 \\
\hline 6 & 15.12 .03 & golssgen. & 2,11 & 126,6 & $-24,5$ & smamluagenonos6 \\
\hline 7 & 28.12 .03 & د. & 2,40 & 144,0 & $-37,1$ & > gamlagengomgs6 \\
\hline 8 & 28.12 .03 & د.लिखिए. & 3,12 & $187,0^{* *}$ & $-37,1$ & ১мamls \\
\hline 9 & 15.01 .04 & s. & 2,38 & 142,7 & $-32,2$ & 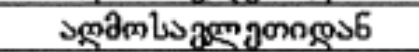 \\
\hline 10 & 03.02 .04 & spaml. & 2.10 & 125,9 & $-24,2$ & 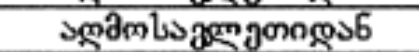 \\
\hline 11 & 03.03 .04 & s. & 1,48 & 88,6 & $-8,9$ & sмamlsagengmogs6 \\
\hline 12 & 05.04 .04 & sceamt. & 0 & - & 2,4 & - \\
\hline 13 & 30.04 .04 & sgaml. & 1,54 & 92,1 & 10,0 & golsagengongos6 \\
\hline 14 & 31.05 .04 & spaml. & 2,01 & 120,4 & 19,8 & golazemanogab \\
\hline 15 & 26.06 .04 & sganl. & 2,17 & 130,2 & 24,6 & gostujenganges \\
\hline 16 & 27.06 .04 & gatusgen. & 2,31 & 138,9 & 30,2 & golugangangas \\
\hline 17 & 27.07 .04 & gologen. & 2,34 & 134,2 & 28,4 & golsagenamgat \\
\hline 18 & 22.08 .04 & smaml. & 2,40 & 144,1 & 37,3 & gutsagenangs6 \\
\hline 19 & 02.09 .04 & sgâl. & 2,10 & $123,1 * * * *$ & 21,2 & golsagengongs \\
\hline 20 & 12.10 .04 & s. & 1,55 & 93,2 & 10,2 & gulazgengong6 \\
\hline 21 & 02.11 .04 & s. & 0 & - & -2.1 & - \\
\hline 22 & 30.11 .04 & دpaml. & 1,57 & 94,3 & $-10,1$ & 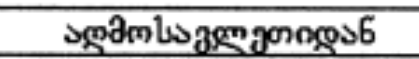 \\
\hline
\end{tabular}

ซj60"

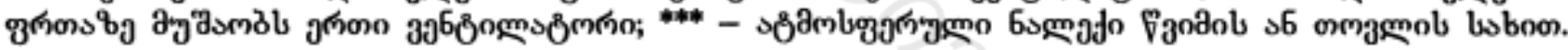

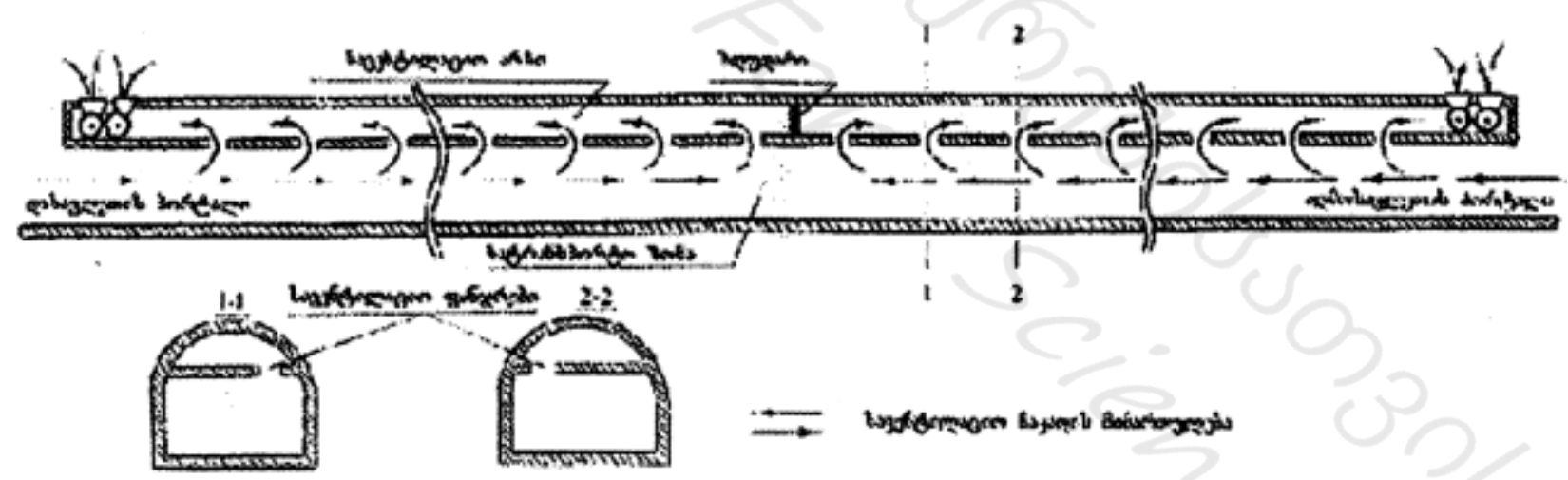

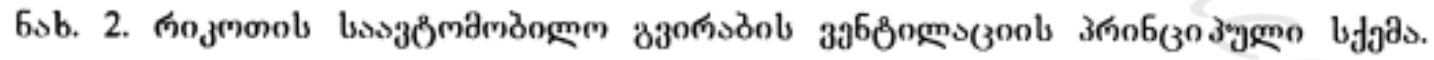

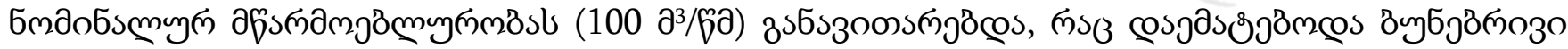

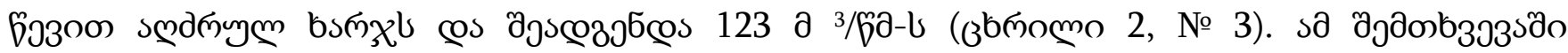

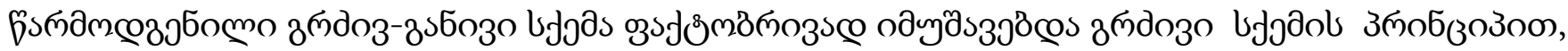

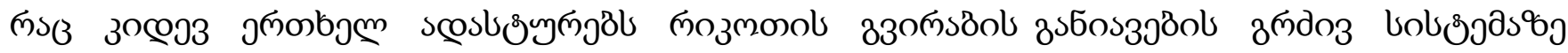

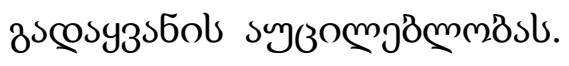

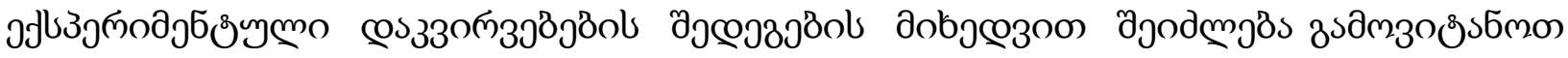

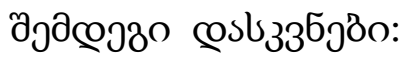

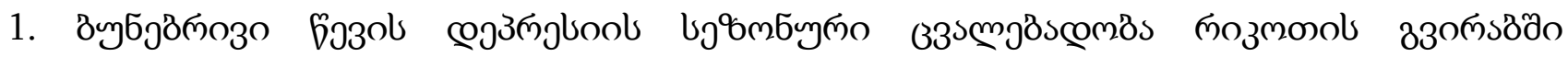

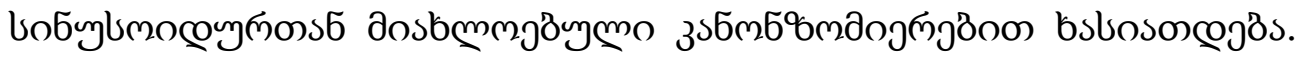




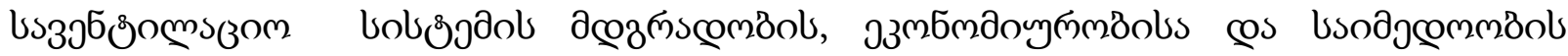

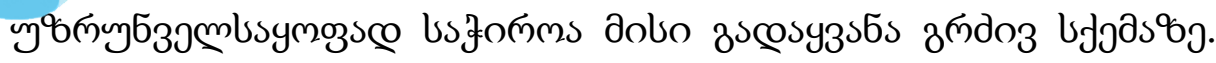

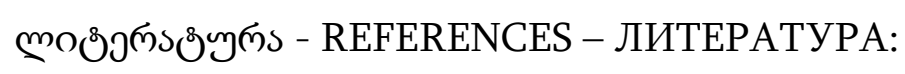

1. Eugenio A. Merzagora.The World's longest Tunnel Page (The Internet).

2. Gunnar Lotsberg. Content of BHR Group Publication. Summary of paper presented at the $^{\text {th }}$ International conference on Aerodynamics and Ventilation of Vehicle Tunnels held in Aosta Valley, Italy, 6-8 October, 1997.

AEROLOGY

\section{SEASONAL CHANGE OF SIZE AND DIRECTION OF NATURAL DRAFT IN RIKOTI ROAD TUNNEL}

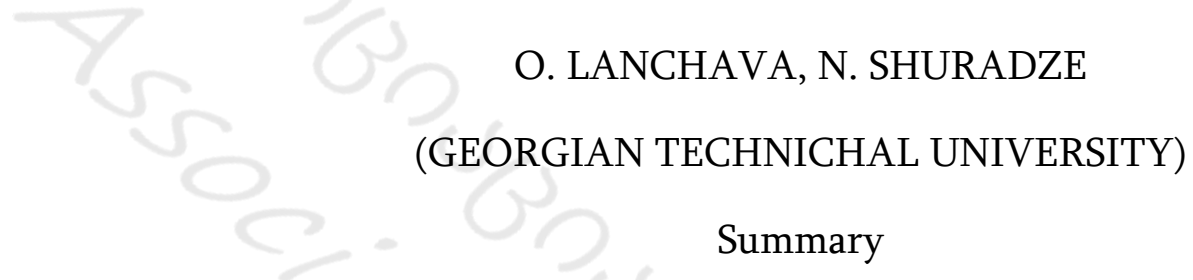

With the purpose of an establishment of character of parameters change of natural draft in Rikoti road tunnel are carried out experimental supervisions The depression of natural draft caused by the climatic factors is reduced to a difference of pressure at portals. The statistical parameters of natural draft and intensity of movement the automobiles are given. There is judged possible expediency of application of the longitudinal circuit of airing in the given tunnel.

АЭРОЛОГИЯ

\section{СЕЗОННОЕ ИЗМЕНЕНИЕ ВЕЛИЧИНЫ И НАПРАВЛЕНИЯ ЕСТЕСТВЕННОЙ ТЯГИ В РИКОТСКОМ АВТОМОБИЛЬНОМ ТОННЕЛЕ}

О. ЛАНЧАВА, Н. ШУРАДЗЕ

(ГРУЗИНСКИЙ ТЕХНИЧЕСКИЙ УНИВЕРСИТЕТ)

Резюме

С целью установления характера изменения параметров естественной тяги в Рикотском автодорожном тоннеле проведены экспериментальные наблюдения. Депрессия естественной тяги, обусловленная климатическими факторами, сводится к разности давлений у порталов. Приведены статистические параметры естественной тяги и интенсивности движения транспорта Делается вывод о возможной целесообразности применения продольной схемы проветривания в данном тоннеле. 